\title{
Importancia dopaminérgica en farmacodinamia de cocaína evaluada por alteraciones de GABA y glutamato en el núcleo accumbens de ratas
}

\author{
Edgar A. Gélvez', Ariel E. Pastrana1, Rafael A. Ulloque²
}

\section{Resumen}

Este trabajo se propuso demostrar la importancia del sistema dopaminérgico en la farmacodinamia de cocaína en el SNC de ratas. Este postulado se verificó evaluando las alteraciones de GABA y glutamato en el núcleo accumbens.

Este enfoque se sustenta en la gran cantidad de evidencias que muestran, consistentemente alteraciones en la actividad dopaminérgica por cocaína. Además, el GABA y el glutamato tienen gran importancia en los efectos agudos y crónicos por cocaína. Es bien conocida la interrelación de GABA y glutamato con la actividad dopaminérgica en el núcleo accumbens.

Se diseñó un estudio por 16 días de administración de cocaína a ratas a la dosis inicial de $30 \mathrm{mg} / \mathrm{kg} / \mathrm{día}$ (IP) con incremento de $5 \mathrm{mg} / \mathrm{kg}$ cada 4 días, a las cuales se les había lesionado la actividad dopaminérgica en el núcleo accumbens con la neurotoxina 6hidroxidopamina en ácido ascórbico. Además, se conformó un grupo basal que recibió estereotáxicamante, en el núcleo accumbens, ácido ascórbico en solución salina 0,9\% y se trató con un volumen correspondiente de solución salina (IP) y, un grupo control, al cual estereotáxicamente, en el núcleo mencionado, se le administró 6-OHDA (en ácido ascórbico), recibiendo posteriormente como tratamiento solución salina (IP). La determinación de los niveles de GABA y glutamato se realizó por métodos enzimáticos.

Se observó que la administración crónica de cocaína produce una disminución significativa en el nivel de GABA en el núcleo accumbens del grupo problema, con relación a los grupos basal y control. En tanto, el nivel de glutamato en el grupo problema mostró un incremento significativo con relación al grupo basal, pero, no con relación al grupo control.

En conclusión, los resultados de este trabajo sostienen la hipótesis del papel importante que tiene la dopamina en la farmacoterapia de cocaína a través de las alteraciones en los niveles de GABA y glutamato o alteraciones en forma directa. Además, la cocaína podría alterar por sí misma la actividad gabaérgica y glutaérgica.

\section{Summary}

The aim of the present study was to investigate the role of dopamine in cocaine pharmacodynamics in rats. To test this hypothesis, we measured GABA and glutamate level changes in the accumbens nucleus. This approach is supported by the preponder-

Estudiante de posgrado de Farmacología, Universidad de Cartagena, Q.E.P.D.

2 Departamento de Farmacología, Universidad de Cartagena, Cartagena, Colombia. 
ance of evidence which has consistently shown alterations in dopaminergic activity after cocaine administration.

Furthermore, GABA and glutamate have considerable importance in cocaine's acute and chronic effects. The relationship between GABA and glutamate with dopaminergic activity in the nucleus accumbens is well-known.

A 16-day study was designed for the administration of cocaine chlorhydrate (IP) at 30 $\mathrm{mg} / \mathrm{kg} /$ day increasing by $5 \mathrm{mg} / \mathrm{kg} /$ day each 4 days to animals which had been stereotaxically injured in the nucleus accumbens with 6-OH-dopamine (6-OHDA) neurotoxin dissolved in ascorbic acid. A base group was formed which received ascorbic acid diluted in $0.9 \%$ saline solution stereotaxically in the nucleus accumbens, and was treated with an equal volume of $0.9 \%$ saline solution (IP). The control group received 6 OHDA (diluted in ascorbic acid) stereotaxically in the nucleus accumbens and was given $0.9 \%$ saline solution as treatment. GABA and glutamate levels were determined by enzymatic assays.

The results of this work show a significant decrease of GABA levels in the group that received chronic cocaine administration in comparison to the base and control group. Glutamate levels were significantly higher in the cocaine group than in the base group, but not significantly different from the control group.

In conclusion, the results of the present work support the idea of the great importance of dopaminergic activity in cocaine pharmacotherapy. This dopamine role could be either through GABA or glutamate level alterations or both. Direct action of increased cocaine level on the so-called reward system could be another explication.

La cocainodependencia y sus concomitantes problemas médico-sociales se han hecho evidentes en muchos países en los últimos años. Estudios epidemiológicos llevados a cabo en los Estados Unidos han revelado que alrededor de 22'000.000 de personas habían utilizado, por lo menos, una vez cocaína y que había, por lo menos, 5'000.000 de usuarios regulares (1). En Colombia, un estudio realizado por el Ministerio de Salud mostró que la prevalencia anual del consumo de cocaína era de 2,8/1.000 en forma global para la población estudiada (2).

Una amplia bibliografía propone un sistema de recompensa o placer el cual sería el sitio de acción de las drogas que causan adicción. Entre las estructuras claves de este sistema está el núcleo accumbens (3-8). En este núcleo existe una interrelación recíproca entre vías dopaminérgicas, gabaérgicas y glutaérgicas. Con relación al entendimiento de los cambios neuronales íntimos relacionados con la cocainodependencia, existe abundante evidencia de la importancia de la actividad dopaminérgica en la aparición y sostenimiento de este fenómeno (914). Con base en este concepto, se han fundamentado múltiples ensayos con fármacos dopaminérgicos (15-23) como parte integral del tratamiento del paciente abusador y adicto a la cocaína.

Sin embargo, el bajo porcentaje de mejoría total plantea la existencia del compromiso de otros sistemas neuronales (24). Investigaciones electrofisiológicas sugieren que, también, puede existir una disminución gabaérgica durante la administración de cocaína $(25,26)$. En nuestro laboratorio, la administración crónica de cocaína produce un descenso de los niveles de GABA en $24 \%$ de las ratas con el núcleo accumbens intacto. De otra manera, también se ha observado un incremento de glutamato de $26 \%$.

El presente trabajo trata de aclarar la importancia de la actividad dopaminérgica en los niveles de GABA y glutamato que producen lesión dopaminérgica con la neurotoxina 6hidroxidopamina (6-OHDA) en el núcleo accumbens de ratas. 


\section{Materiales y métodos}

Se utilizaron ratas machos, albinos, SpragueDawley, de aproximadamente 250-300 g, adquiridas a través del Bioterio del Instituto Nacional de Salud.

Se diseñó un estudio por 16 días. Este tuvo un grupo basal, un grupo control y un grupo problema, cada uno de ocho animales. El grupo control basal recibió un volumen de $2 \mathrm{~mL}$ de solución de ácido ascórbico al $0,1 \%$ en solución salina, en el núcleo accumbens, mediante estereotaxia bilateral de acuerdo con las coordenadas: A-P $+3 \mathrm{~mm} ; \mathrm{L} \pm 1,4 \mathrm{~mm} ; V 7,4$ $\mathrm{mm}$ (27). El grupo control y el grupo problema recibieron, en la misma forma, $2 \mathrm{~mL}$ de solución de 6-hidroxidopamina $(6 \mathrm{mg} / \mathrm{mL})$ disuelta en solución de ácido ascórbico.

Tres días después de realizado lo anterior, se administró solución salina (IP) a los grupos basal y control, y clorhidrato de cocaína (Merck, adquirida del Fondo Nacional de Estupefacientes, Ministerio de Salud) por la misma vía al grupo problema a la dosis inicial de $30 \mathrm{mg} / \mathrm{kg} / \mathrm{dí}$ a con incremento de $5 \mathrm{mg} / \mathrm{kg}$ cada 4 días, durante el período del estudio.

Un día después de terminada la administración de la solución salina a los grupos basal y control y del clorhidrato de cocaína al grupo problema, los animales se sacrificaron por decapitación; se les removió el cerebro del cual se hicieron cortes coronales de $2 \mathrm{~mm}$ de espesor siguiento la topografía del atlas de G. Paxinos, $2 \mathrm{~mm}$ anterior al quiasma óptico. En estas cortes se identifica el área del núcleo accumbens, el cual se aisló, maceró y centrifugó. Con el sobrenadante, se determinaron los niveles de GABA y de glutamato con la ayuda de los métodos enzimáticos de la gabasa y glutámico deshidrogenasa, respectivamente (28-31).

La significancia estadística de la diferencia entre los grupos basal, control y problema se estableció de acuerdo con la prueba de Newman-Keuls (32).

\section{Resultados}

El cuadro 1 muestra los niveles de GABA y glutamato en el núcleo accumbens de ratas en micromoles por gramo de tejido en el grupo basal que recibió extereotáxicamente solución de ácido ascórbico y los grupos control y problema que recibieron 6-hidroxidopamina en solución salina en el mismo núcleo. Durante el período de estudio los dos primeros grupos recibieron solución salina normal y el grupo problema recibió clorhidrato de cocaína a las dosis indicadas (IP) .

Se observó una diferencia estadísticamente significativa entre sí $(q>4,61 ; p<0,01)$ en los niveles de GABA de los grupos basal, control y problema. En los niveles de glutamato, se

Cuadro 1. Niveles de GABA y glutamato en el núcleo accumbens de ratas tratadas con cocaína.

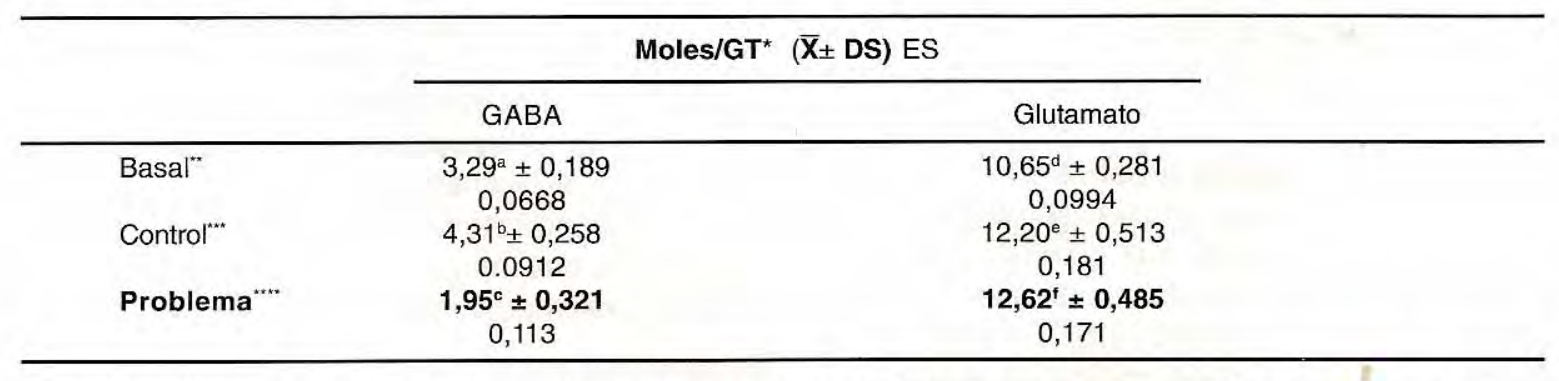

* Cada valor es el promedio, la desviación estándar y el error estándar de ocho animales, en triplicado cada uno.

** Solución de ácido ascórbico más solución salina IP.

*** 6-hidroxidopamina, $2 \mathrm{~mL}(6 \mathrm{mg} / \mathrm{mL})$ más solución salina IP.

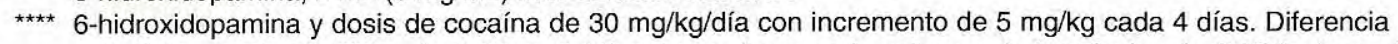
estadísticamente signifitiva $(q>4,61 ; p<0,01)$ entre: $a$, b y $c$ en la columna de los niveles de $G A B A ; d$ y e, $d$ y $f$ en la columna de los niveles de glutamato. 
observó una diferencia estadísticamente significativa $(q>4,61 ; p<0,01)$ entre los grupos basal y control, y entre el basal y el problema.

\section{Discusión}

Los resultados del presente trabajo, donde se lesionaron los terminales dopaminérgicos en el núcleo accumbens con 6-OHDA, muestran que la administración crónica de cocaína produce disminución estadísticamente significativa ( $q>$ $4,61 ; p<0,01)$ con relación a los grupos basal y control en los niveles de GABA. Estos resultados están de acuerdo con los antecedentes y planteamientos mencionados. Lo anterior podría explicarse teniendo en cuenta que la lesión por 6-OHDA produce una supersensibilidad de los receptores dopaminérgicos $D_{2}(33)$ en neuronas gabaérgicas $y$, al existir un reducto de terminales dopaminérgicos sin lesión (34), la administración de cocaína produciría un disparo dopaminérgico exagerado sobre la actividad gabaérgica. Este hallazgo sustenta el concepto que la cocaína afecta directamente la actividad gabaérgica, regulando los receptores $D_{2}$ sobre estas neuronas (35-37).

Los niveles de glutamato en el grupo problema se elevaron significativamente con relación al grupo basal $(q>4,61 ; p<0,01)$, pero, no con relación al grupo control. Además, es de anotar la diferencia significativa entre los grupos basal y control; los niveles de glutamato fueron mayores $(q>4,61 ; p<0,01)$. Esto confirma la interregulación dopamina-glutamato. Lo anterior se sustenta teniendo en cuenta que la dopamina frena la actividad (liberación) glutaérgica en este núcleo (38-40).

Nuestros resultados sustentan la idea de que, al lesionar los terminales dopaminérgicos, la cocaína no altera los niveles de glutamato ya incrementados por esta lesión, teniendo en cuenta que la dopamina frena la liberación de glutamato, especialmente, en las proyecciones de axón largo del hipocampo y la amígdala al núcleo accumbens $(41,42)$.

Este sería el caso de la administración de cocaína donde los niveles de glutamato tienden a mantenerse elevados. Sin embargo, el animal crónico tiende a un estado depresivo cuyo sustrato neurobioquímico sería exacta-mente la disminución de la actividad dopami-nérgica. Se conoce de la capacidad excitatoria directa por la dopamina (43-46).

Es de importancia considerar los efectos agudos y crónicos por cocaína en animales sin lesión, sobre los niveles de GABA y glutamato. Se especificó anteriormente la alteración de estos dos neurotransmisores por cocaína en el núcleo accumbens, apoyando así la noción de la importancia que ellos tendrían en los efectos agudos y crónicos por esta droga.

Todo lo anterior, tomado en conjunto, sostiene la hipótesis que la dopamina juega un papel importante en la neurobioquímica y en los efectos farmacológicas por la cocaína, en forma directa o a través de alteraciones que produciría sobre neurotransmisores involucrados en el fenómeno de la cocainodependencia.

\section{Conclusiones}

La administración crónica de cocaína produce una disminución significativa en los niveles de GABA en el núcleo accumbens con lesión dopaminérgica.

La administración crónica de cocaína no produce variación en los niveles de glutamato en el núcleo accumbens con lesión dopaminérgica.

La cocaína afectaría directamente neuronas gabaérgicas y no a través de mecanismos dopaminérgicos.

La cocaína afecta la actividad glutaérgica a través de mecanismos dopaminérgicos.

Las observaciones de esta investigación demuestran la importancia del sistema dopaminérgico sobre la actividad gabaérgica y glutaérgica. Estos resultados sugieren que la cocaína produciría sus efectos a través de mecanismos, principalmente, dopaminérgicos; sin embargo, existirían otros mecanismos involucrados. Estos resultados podrían ser de gran ayuda para el desarrollo de nuevos enfoques experimentales y terapéuticos en el manejo integral de la cocainodependencia en el humano. 


\section{Agradecimientos}

A la Universidad de Cartagena y Colciencias por la evaluación y apoyo financiero de esta investigación.

Al Fondo Nacional de Estupefacientes del Ministerio del Salud por el suministro de la cocaína.

\section{Referencias}

1. Clayton RR. Cocaine use in the United States: in a blizzard or just being snowed? In: Kozel NJ, Adams $\mathrm{EH}$, eds. Cocaine use in America: epidemiologic and clinical perspectives. Washington, D. C.: U.S. Government Printing Office, 1985;61:8-34.

2. Posada JV, Torres Y. Patrones de consumo de cocaína. En: Estudio nacional de salud mental y consumo de sustancias psicoactivas, Colombia,1993. Santa Fe de Bogotá: Ministerio de Salud, 1994:25063.

3. Barnes DM. The biological tangle of drug addiction. Science 1988;241:415-6.

4. Koob GF. Drugs of abuse: anatomy, pharmacology and function of reward pathways. TIPS 1992;13 (5):177-84.

5. Pijnenburg AJ, Van Rossum JM. Stimulation of locomotor activity following injection of dopamine into the nucleus accumbens. J Pharm Pharmacol 1973; 25:1003-5.

6. Makanjuola RA, Dow RC, Aschcroft GW. Behavioral responses to stereotactically controlled injections of monoamine neurotransmitters into the accumbens and caudate-putamen nuclei. Psychopharmacology 1980;71:227-35.

7. Delfs MJ, Schreiber L, Kelley AE. Microinjection of cocaine into the nucleus accumbens elicits locomotor activity in the rat. J Neurosci 1990;10:303-10.

8. Chang DM, Sawyer SF, Lee RS, Woodward DJ. Electrophysiological and pharmacological evidence for the role of the nucleus accumbens in cocaine self-administration in freely moving rats. $J$ Neurosci 1994; 13(3):1224-44.

9. Goeders NE, Smith JE. Cortical dopaminergic involvement in cocaine reinforcement. Science 1983; 221:773-5.

10. Di Chiara G, Imperato A. Drugs abused by humans preferentially increase synaptic dopamine concentrations in the mesolimbic system of freely moving rats. Proc Natl Acad Sci 1988;35:5274-8.

11. Koob GF, Bloom FE. Cellular and molecular mechanisms of drug dependence. Science 1988;242:71523.

12. Hurd Y, Ungerstedt $U$. Cocaine: an in vivo micro-dialysis evaluation of its acute action on dopamine transmission in rat striatum. Synapse 1989;3:48-54.
13. Kuhar MJ, Ritz MC, Boja JM. The dopamine hypothesis of the reinforcing properties of cocaine. TIPS 1991;14(7):299-302.

14. Caine SB, Koob GF. Effects of mesolimbic dopamine depletion on responding maintained by cocaine and food. J Exp Anal Behav 1994;61:213-21.

15. Baxter LR. Desipramine in the treatment of hypersomnolence following abrupt cessation of cocaine use. Am J Psychiatry 1983;140:1525-6.

16. Gawin FH. New uses of antidepressant in cocaine abuse. Psychosomatics 1986;27:24-9.

17. Giannini AJ, Malone DA, Giannini MC, Price WA, Loiselle RH. Treatment of depression in chronic cocaine and phencyclidine abuse with desipramine. J Clin Pharmacol 1986;26:211-4.

18. Giannini AJ, Baumgartel P, DiMarzio LR. Bromocriptine therapy in cocaine withdrawal. J Clin Pharmacol 1987;27:267-70.

19. Giannini AJ, Billett W. Bromocriptine-desipramine protocol in treatment of cocaine addiction. J Clin Pharmacol 1987;27:549-54.

20. Dackis CA, Gold MS, Sweeney DR, Byron JP, Climko R. Single-dose bromocriptine reverses cocaine craving. Psychiatry Res 1987;20:261-4.

21. Weiss RD. Relapse to cocaine abuse after initiating desipramine treatment. JAMA 1988;260(17):2545-6.

22. Gawin FH, Klebert HD, Byck R, Rounsaville BJ, Kosten TR, Jatlow PI, et al. Desipramine facilitation of initial cocaine abstinence. Arch Gen Psychiatry 1989;46:117-21.

23. Witkin JM. Pharmacotherapy of cocaine abuse: Preclinical development. Neurosci Biobehav Rev 1994;18(1):121-42.

24. Gawin FH, Ellinwood EH. Cocaine and ather stimulants. N Engl J Med 1988;318:1173-82.

25. Guerrero RF. Precipitación del síndrome convulsivo del lóbulo temporal por cocaína en humano. Ciencia Tecnología y Educación, Universidad de Cartagena. 1986;4(1):9-21.

26. Guerrero RF, Guerrero AR. Cambios de excitabilidad neuronal hipocampal durante la administración de cocaína. Acta Médica de Cartagena 1991;3(1):2330.

27. Paxinos G, Watson $\mathbf{C}$. The rat brain in stereotaxic coordinates. Second edition. San Diego: Academic Press, 1986; figure 10 and 80.

28. Graham LT, Aprison MH. Fluorometric determination of aspartate, glutamate and gamma-amino-butyrate in nerve tissue using enzymic methods. Analytical Biochem 1966;15:487-97.

29. Fahn S, Côté LJ. Regional distribution of gammaaminobutyric acid (GABA) in brain of the Rhesus monkey. J Neurochem 1968;15:209-13. 
30. Kravitz EA, Kuffler SW, Potter DD, Van Gelder NM. Gamma-aminobutyric acid and other blocking compounds in crustaceae. J Neurophysiol 1963;26: 729-38.

31. Grahan LT, Werman R, Aprison MH. Microdetermination of glutamate in single cat spinal roots. Life Sci 1965;4(10):1085-90.

32. Tallarida RJ, Murray RB. Newman-Keuls test. In: Manual of pharmacologic calculation with computer programs. Second edition. New York: SpringerVerlag, 1987:121-5.

33. Marcotte ER, Sullivan RM, Mishra RK. Striatal Gproteins: effects of unilateral 6-hidroxydopamine lesions. Neurosci Lett 1994;169(1):195-8.

34. Pettit HO, Ettenberg A, Bloom FE, Koob GF. Destruction of dopamine in the nucleus accumbens selectively attenuates cocaine but not lesion self-administration in rats. Psychopharmacology 1984;84:16773.

35. Gale K. Catecholamine-independent behavioral and neurochemical effects of cocaine in rat. In: Sharp $\mathrm{CM}$, ed. Mechanisms of tolerance and dependence. Washington, D. C.: U.S. Government Printing Office, 1984;54:223-31.

36. Reid MS, O'Connor WT, Herrera-Maeschizt M, Ungerstedt $U$. The effects of intranigral GABA and dynorphin $A$ injections on striatal dopamine and GABA release: evidence that dopamine provides inhibitory regulation of striatal GABA neurons via $D_{2}$ receptors. Brain Res 1990;519:255-60.

37. Karler R, Calder LD, Thai LH, Bedingfield $\mathbf{B}$. The dopaminergic, glutamatergic, GABAergic bases for the action of amphetamine and cocaine. Brain Res $1995 ; 671: 100-4$.
38. Linderfors $\mathbf{N}$, Ungerstad $\mathbf{U}$. Bilateral regulation of glutamate tissue and extracellular level in caudateputamen by midbrain dopamine neurons. Neurosci Lett 1990;31(2):248-52.

39. Karler R, Calder LD. Excitatory aminoacids and the action of cocaine. Brain Res 1992;582:143-6.

40. Karler R, Calder LD, Thai LH, Bedingfield J. A dopaminergic-glutaergic basis for the action of amphetamine and cocaine. Brain Res 1994;658:8-14.

41. De France JF, Marchand JE, Stanley JC, Sikes RW, Chronister RB. Convergence of excitatory amygdaloid and hippocampal input in the nucleus accumbens septi. Brain Res 1980;185:183-6.

42. Robinson TG, Beart PM. Excitant aminoacid projections from rat amygdala and thalamus to the nucleus accumbens. Brain Res Bull 1988;20:467-71.

43. Pijnenburg AJ, Van Rossum JM. Stimulation of locomotor activity following injection of dopamine into the nucleus accumbens. J Pharm Pharmacol 1973; 25:1003-5.

44. Jones DL, Mogenson GJ, Wu M. Injections of dopaminergic, cholinergic, serotonergic and gabaergic drugs into the nucleus accumbens: effects on locomotor activity in the rat. Neuropharmacology 1981;20:29-37.

45. Stewart J. Neurobiology of conditioning to drugs of abuse. In: Kalivas PW, Samson $\mathrm{HH}$, eds. The neurobiology of drug and alcohol addiction. New York: Annals of the New York Academy of Sciences 1992; 654:335-46.

46. Willins DL, Narayanan S, Wallage LJ, Uretsky N. The role of dopamine and AMPA/kainate receptors in the nucleus accumbens in the hypermotility response to MK-801. Pharmacol Biochem Behav 1993;46:881-7. 